\title{
Little Toe Osteosarcoma: A Rare Case
}

\author{
Sam Hadjialiloo Sami, ${ }^{1}$ Tina Shoshtarizadeh, ${ }^{1}$ Hajar Zekavat, ${ }^{1}$ and Mehrdad Bahrabadi ${ }^{1,}{ }^{*}$ \\ ${ }^{1}$ Bone and Joint Reconstruction Research Center, Shafa Orthopedic Hospital, Iran University of Medical Sciences, Tehran, IR Iran \\ "Corresponding author: Mehrdad Bahrabadi, Bone and Joint Reconstruction Research Center, Shafa Orthopedic Hospital, Iran University of Medical Sciences, Tehran, IR Iran. \\ Tel: +98-2133542000-8, Fax: +98-2133542020, E-mail: mehrdad.bahrabadi@gmail.com
}

Received 2016 May 31; Revised 2016 August 30; Accepted 2016 September 16.

\begin{abstract}
Introduction: Osteosarcoma is the second most common primary bone tumor, however, osteosarcoma of the foot and especially of the toe phalanges are very rare.

Case Presentation: The current report includes the radiological, pathological, and short term clinical results of a conventional high grade osteosarcoma of the little toe in a 17-year-old adolescent. He referred to the center with a painful swelling at posterolateral aspect of his left fifth toe. Imaging studies were consistent with an aggressive lesion. Biopsy confirmed the diagnosis of conventional osteosarcoma.

Conclusions: In case of osteosarcoma of little toe proximal phalanx in patients with a bone destructive lesion, malignancy should always be considered.
\end{abstract}

Keywords: Osteosarcoma, Little Toe Proximal Phalanx, Conventional

\section{Introduction}

Osteosarcoma is the second most common primary malignant bone tumor, with a peak incidence in the second decade of life (1). Usually, it originates at metaphysis of long bones around the knee (1). The largest subtype of osteosarcoma is conventional. It has the same skeletal distribution and age range of the osteosarcoma.

Primary osteosarcoma of foot bones is very rare (1-3). It represents approximately $0.5 \%$ of all osteosarcomas (2). Primary osteosarcoma originating in a toe phalanx is even more exceptional. The first case of toe phalanx osteosarcoma was reported in 1988 (4). Another case was reported in 1995 (5). These two cases involved great toe phalanges. The first reported case of small cell osteosarcoma of a toe phalanx was reported in 2010 (6). It was a small cell osteosarcoma and not a conventional one. There was another case report of an osteosarcoma of the middle and distal phalanges of the little toe, but this case did not show osteoid in its histopathology exam (7). To the best of authors' knowledge, the current case was the first reported case of a conventional osteosarcoma originating from little toe proximal phalanx in the English literature.

\section{Case Presentation}

The study was approved by the ethic board of the institution and the patient's parents signed a written consent to report the case. A17-year-old male patient was admitted to the hospital complaining of pain and swelling of his left fifth toe. This problem had been present for 10 months before his visit. The swelling had hard consistency, and was located at the dorsolateral aspect of little toe. The mass was slightly tender to touch. Family history and past medical history were unremarkable. His physical exam was normal except the presence of the mass. Laboratory tests were within normal range. Radiography of the foot showed a dense lesion with destroyed cortices of the proximal phalanx of the fifth toe. Embedded in radio-opaque area, there were small areas of radiolucency (Figure 1A). There was soft tissue swelling around the fifth toe, but no periosteal reaction around the lesion. All joints of the fifth toe were radiologically normal. Technetium bone scan was performed, which showed an increased tracer uptake. Magnetic resonance imaging(MRI) of the lesion showed low signal lesion on $\mathrm{T} 1$ and high signal lesion with a soft tissue mass on T2 (Figure 1B - 1C).

Considering the history, physical exam, and imaging studies, which were in favor of an aggressive or malignant lesion, and since malignant tumors were in the differential diagnostic list, it was considered to perform an incisional biopsy to reach the final diagnosis. The biopsy was consistent with conventional high grade osteosarcoma (Figure 2).

The patient received pre-operative chemotherapy, with doxorubicin, and cisplatin, surgery, and post-operative 

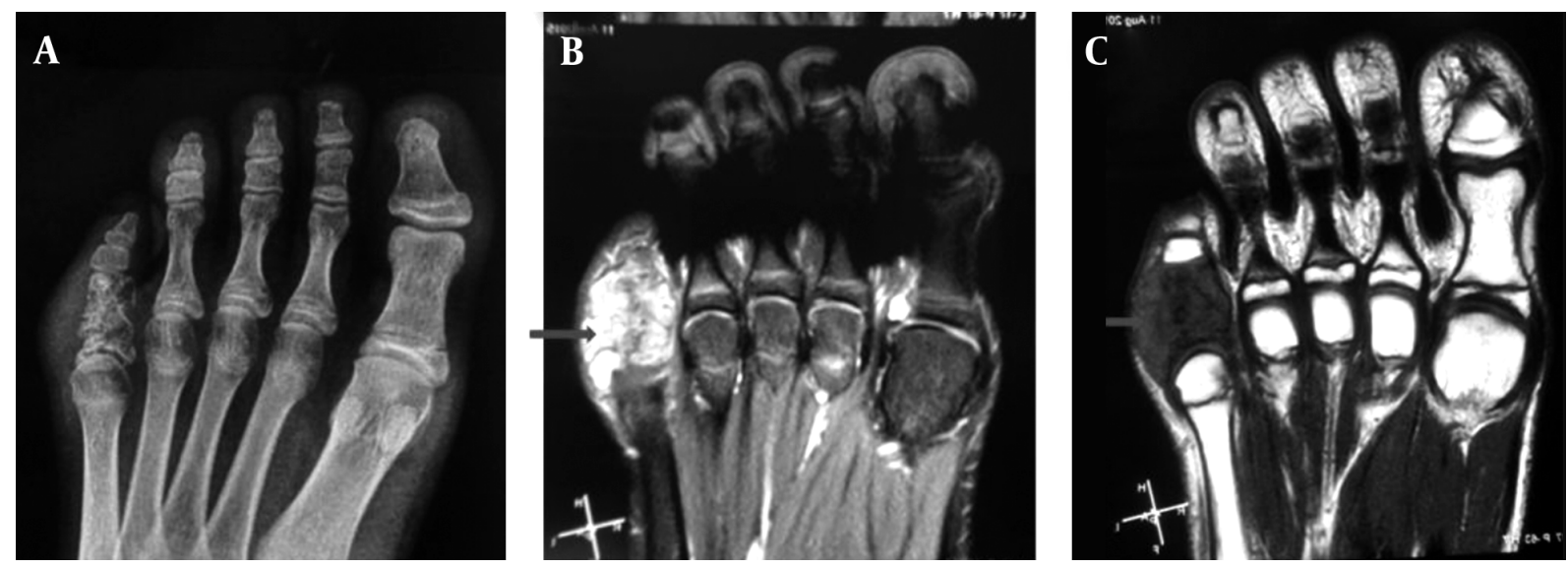

Figure 1. A, Plain radiography of the left foot showing a radio-opaque lesion with destroyed cortices of the proximal phalanx of the fifth toe; B, and C, magnetic resonance imaging of the left foot demonstrating a lesion with high signal intensity on T2 and low signal on T1. On both views, soft tissue component of the tumor is visible (arrows).
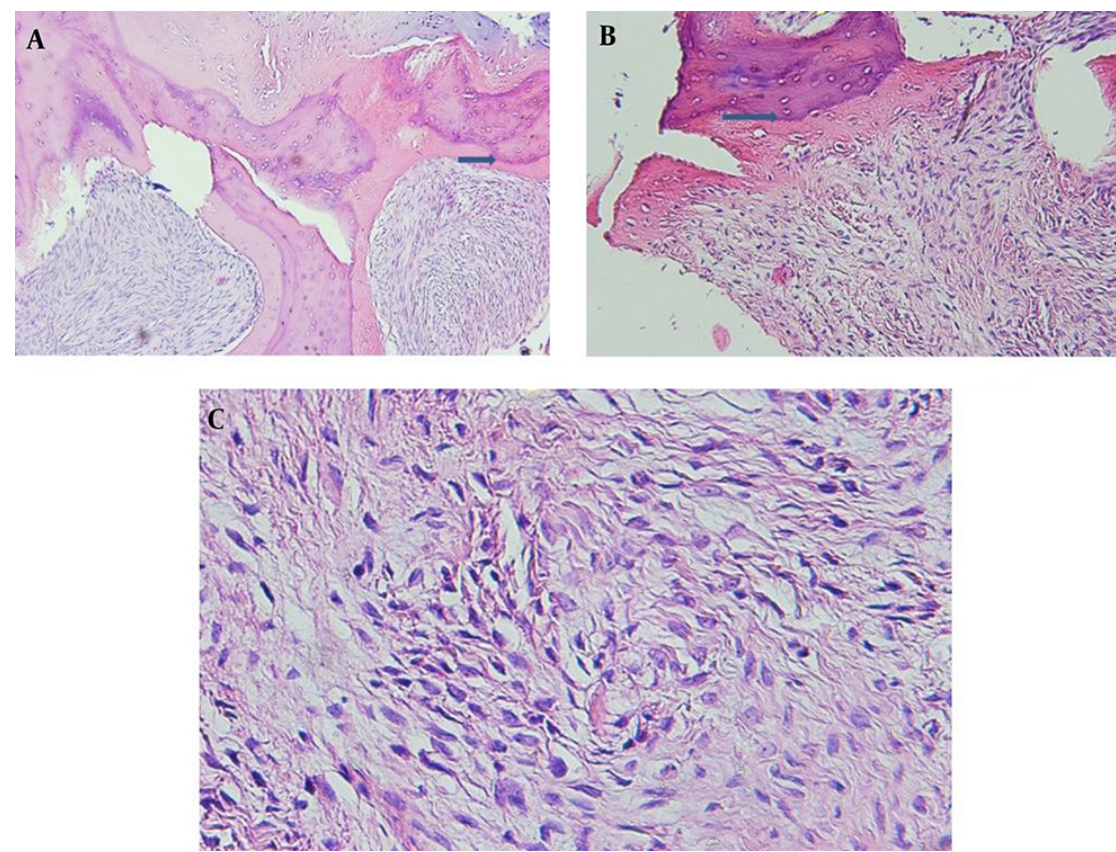

Figure 2. A and B, Photomicrographs of the resected tumor showing trabecular bone surrounded by spindle cells producing osteoid (arrows) ( $\mathrm{H}$ and $\mathrm{E} x \mathrm{4}$ ) of Figure 1 and ( $\mathrm{H}$ and E x 10) of Figure 2C; , diffuse proliferation of short spindle mesenchymal cells with loose arrangement, atypia and large vesicular nuclei (H and E x 40).

chemotherapy with the same regimen. For the surgical procedure, it was decided to perform an amputation of the fifth ray. There was a good response to chemotherapy, evidenced by a necrosis rate of more than $90 \%$.

The postoperative visits of the patient were scheduled at two and six weeks, three and six months. The last visit was nine months after the amputation. He did not show any sign of tumor recurrence, or any distant metastasis. He was off the chemotherapy drugs.

\section{Discussion}

Primary osteosarcoma of the foot, especially the ones involving the toe phalanges, is quite rare; therefore, any case of them may be worth reporting. From 1911 to 1992, only 12 osteosarcomas of foot origin were detected which made only $0.6 \%$ of all osteosarcomas diagnosed in Rizzoli institute (3). In Mayo clinic, from 1941 to 1991, 60 patients were diagnosed with osteosarcoma of the foot and 
only four of the patients had toe phalanx osteosarcoma (8). The incidence of histological subtypes in their series of 52 foot osteosarcomas was in accordance with that of encountered in osteosarcoma in general. To the best of authors' knowledge, the current study patient was the first reported case of little toe proximal phalanx osteosarcoma in the English literature.

Clinical features of conventional osteosarcoma include pain and swelling. It could have a large soft tissue mass if diagnosis is delayed. Other symptoms are rare. In small tubular bones, the same symptoms can occur, but since this area is not a common place for osteosarcoma, diagnosis can be difficult without a biopsy. In addition, according to the reported patients in Mayo clinic, diagnosis can be delayed (8). It is unclear whether this delay is due to slow growing potential of the tumor, or other reasons are behind that (8). This is in contrast with conventional osteosarcoma, where the diagnosis is usually made within months of the symptoms onset.

Foot osteosarcoma is usually not a good candidate for limb-salvage surgery due to the poor compartmentalization of the tumor in the foot, and the need to amputation to achieve adequate oncologic margins (8). Therefore, ray amputation was considered as the surgical procedure for the current study patient. In addition, losing a ray, especially the fifth ray is not a big problem to walking or even moderate sport activities. Shoe filler in these cases might be needed for proper shoe filling and better walking quality. Conventional osteosarcoma of little toe proximal phalanx, should be considered in patients with a bone destruc- tive lesion to avoid delay in diagnosis and applying appropriate treatment even though it is very rare.

\section{Footnote}

Authors' Contribution: Diagnosis and treatment, Sam Hadjialiloo Sami and Mehrdad Bahrabadi; manuscript preparation and revision, Sam Hadjialiloo Sami, Tina Shoshtarizadeh, Hajar Zekavat and Mehrdad Bahrabadi.

\section{References}

1. Amini M, Colacecchi C.An unusual case of primary osteosarcoma of the talus. Clin Orthop Relat Res. 1980(150):217-9. [PubMed: 6933030].

2. Lee EY, Seeger LL, Nelson SD, Eckardt JJ. Primary osteosarcoma of a metatarsal bone. Skeletal Radiol. 2000;29(8):474-6. [PubMed:11026717].

3. Biscaglia R, Gasbarrini A, Bohling T, Bacchini P, Bertoni F, Picci P. Osteosarcoma of the bones of the foot-an easily misdiagnosed malignant tumor. Mayo Clin Proc. 1998;73(9):842-7. doi:10.4065/73.9.842.[PubMed: 9737220].

4. Mirra JM, Kameda N, Rosen G, Eckardt J. Primary osteosarcoma of toe phalanx: first documented case. Review of osteosarcoma of short tubular bones. Am J Surg Pathol. 1988;12(4):300-7. [PubMed: 3281481].

5. Chan YF, Llewellyn H. Sclerosing osteosarcoma of the great toe phalanx in an 11-year-old girl. Histopathology. 1995;26(3):281-4. [PubMed: 7797207].

6. Posthumadeboer J, Graat HC, Bras J, Saouti R. Small cell osteosarcoma of a toe phalanx: a case report and review of literature.J Orthop Surg Res. 2010;5:36. doi: 10.1186/1749-799X-5-36. [PubMed: 20525255].

7. Matsunobu T, Bekki H, Harimaya K, Matsumoto Y, Endo M, Yoshitake K, et al. Osteosarcoma of the middle and distal phalanges of the little toe with a cancerous ulcer. IJCRI. 2016;7(3):185-9.

8. Choong PF, Qureshi AA, Sim FH, Unni KK. Osteosarcoma of the foot: a review of 52 patients at the Mayo Clinic. Acta Orthop Scand. 1999;70(4):361-4. [PubMed: 10569266]. 